\title{
Applications of Borel Distribution for a New Family of Bi-Univalent Functions Defined by Horadam Polynomials
}

\author{
S. R. SWAMY ${ }^{1}$, ALINA ALB LUPAŞ ${ }^{2}$, ABBAS KAREEM WANAS $^{3}$, J. NIRMALA ${ }^{4}$ \\ ${ }^{1}$ Department of Computer Science and Engineering, RV College of Engineering, \\ Bengaluru - 560 059, Karnataka, INDIA \\ ${ }^{2}$ Department of Mathematics and Computer Science, University of Oradea, \\ str. Universitatii nr. 1, 410087 Oradea, ROMANIA \\ ${ }^{3}$ Department of Mathematics, College of Science, \\ University of Al-Qadisiyah, Al Diwaniyah, Al-Qadisiyah, IRAQ \\ ${ }^{4}$ Department of Mathematics, Maharani's Science College for Women, \\ Bengaluru - 560 001, INDIA
}

\begin{abstract}
In this paper, by making use of Borel distribution we introduce a new family $\mathcal{G}_{\Sigma}(\delta, \gamma, \lambda, \tau, r)$ of normalized analytic and bi-univalent functions in the open unit disk $U$, which are associated with Horadam polynomials. We establish upper bounds for the initial Taylor-Maclaurin coefficients $\left|a_{2}\right|$ and $\left|a_{3}\right|$ of functions belonging to the analytic and bi-univalent function family which we have introduced here. Furthermore, we establish the Fekete-Szegö problem of functions in this new family.
\end{abstract}

Key-Words: - Bi-univalent function, Bazilevič function, $\lambda$-Pseudo-starlike function, Borel distribution, Horadam polynomials, Upper bounds, Fekete-Szegö problem.

Received: May 28, 2021. Revised: October 31, 2021. Accepted: November 12, 2021. Published: December 1, 2021.

\section{Introduction}

Indicate by $\mathcal{A}$, the collection of analytic functions in the open unit disk $U=\{z \in \mathbb{C}:|z|<1\}$ that have the form:

$$
f(z)=z+\sum_{n=2}^{\infty} a_{n} z^{n}
$$

Further, assume that $S$ stands for the subcollection of the set $\mathcal{A}$ containing of functions in $U$ satisfying (11) which are univalent in $U$.

A function $f \in \mathcal{A}$ is called Bazilevič function in $U$ if (see [26])

$$
\operatorname{Re}\left\{\frac{z^{1-\gamma} f^{\prime}(z)}{(f(z))^{1-\gamma}}\right\}>0, \quad(z \in U, \gamma \geq 0)
$$

A function $f \in \mathcal{A}$ is called $\lambda$-pseudo-starlike function in $U$ if (see [7])

$$
\operatorname{Re}\left\{\frac{z\left(f^{\prime}(z)\right)^{\lambda}}{f(z)}\right\}>0, \quad(z \in U, \lambda \geq 1) .
$$

The elementary distributions such as the Poisson, the Pascal, the Logarithmic, the Binomial, the beta negative binomial have been partially studied in Geometric Function Theory from a theoretical point of view (see for example [6, 11, 22, 24, 40]).

Very recently, Wanas and Khuttar [42] introduced the following power series whose coefficients are probabilities of the Borel distribution:

$$
\begin{gathered}
\mathcal{M}(\tau, z)=z+\sum_{n=2}^{\infty} \frac{(\tau(n-1))^{n-2} e^{-\tau(n-1)}}{(n-1) !} z^{n} \\
(z \in U ; 0<\tau \leq 1) .
\end{gathered}
$$

We note by the familiar Ratio Test that the radius of convergence of the above series is infinity. 
The linear operator $\mathcal{B}_{\tau}: \mathcal{A} \longrightarrow \mathcal{A}$ is defined as follows (see [42])

$$
\begin{gathered}
\mathcal{B}_{\tau} f(z)=\mathcal{M}(\tau, z) * f(z)= \\
z+\sum_{n=2}^{\infty} \frac{(\tau(n-1))^{n-2} e^{-\tau(n-1)}}{(n-1) !} a_{n} z^{n} z \in U,
\end{gathered}
$$

where $(*)$ indicate the Hadamard product of two series.

According to the Koebe One-Quarter Theorem [10] every function $f \in S$ has an inverse $f^{-1}$ defined by $f^{-1}(f(z))=z,(z \in U)$ and $f\left(f^{-1}(w)\right)=w,\left(|w|<r_{0}(f), r_{0}(f) \geq \frac{1}{4}\right)$, where

$$
\begin{gathered}
g(w)=f^{-1}(w)=w-a_{2} w^{2}+\left(2 a_{2}^{2}-a_{3}\right) w^{3}- \\
\left(5 a_{2}^{3}-5 a_{2} a_{3}+a_{4}\right) w^{4}+\cdots .
\end{gathered}
$$

A function $f \in \mathcal{A}$ is said to be bi-univalent in $U$ if both $f$ and $f^{-1}$ are univalent in $U$. Let $\Sigma$ stands for the class of bi-univalent functions in $U$ given by (1).

Srivastava et al. [29] have actually revived the study of analytic and bi-univalent functions in recent years it was followed by such works as those by Bulut [8], Adegani et al. [2], Güney et al. [12], Srivastaya and Wanas [30] and others (see, for example [9, 16, 19, 23, 25, 27, 31, 34, 35, 36, 37, 38, 44]).

We notice that the class $\Sigma$ is not empty. For example, the functions $z, \frac{z}{1-z},-\log (1-z)$ and $\frac{1}{2} \log \frac{1+z}{1-z}$ are members of $\Sigma$. However, the Koebe function is not a member of $\Sigma$. Until now, the coefficient estimate problem for each of the following Taylor-Maclaurin coefficients $\left|a_{n}\right|,(n=3,4, \cdots)$ for functions $f \in \Sigma$ is still an open problem.

Let the functions $f$ and $g$ be analytic in $U$. We say that the function $f$ is subordinate to $g$, if there exists a Schwarz function $\omega$ analytic in $U$ with $\omega(0)=0$ and $|\omega(z)|<1(z \in U)$ such that $f(z)=g(\omega(z))$. This subordination is denoted by $f \prec g$ or $f(z) \prec g(z)(z \in U)$. It is well known that (see [21]), if the function $g$ is univalent in $U$, then $f \prec g$ if and only if $f(0)=g(0)$ and $f(U) \subset g(U)$.

The Horadam polynomials $h_{n}(r)$ are defined by the following repetition relation (see [14]):

$$
\begin{aligned}
& h_{n}(r)=\operatorname{prh}_{n-1}(r)+q h_{n-2}(r) \\
& (r \in \mathbb{R}, n \in \mathbb{N}=\{1,2,3, \cdots\}),
\end{aligned}
$$

with $h_{1}(r)=a$ and $h_{2}(r)=b r$, for some real constant $a, b, p$ and $q$. The characteristic equation of repetition relation (3) is $t^{2}-p r t-q=0$. This equation has two real roots $x=\frac{p r+\sqrt{p^{2} r^{2}+4 q}}{2}$ and $y=\frac{p r-\sqrt{p^{2} r^{2}+4 q}}{2}$.

Remark 2.1. By selecting the particular values of $a, b, p$ and $q$, the Horadam polynomial $h_{n}(r)$ reduces to several polynomials. Some of them are illustrated below:

1. Taking $a=b=p=q=1$, we obtain the Fibonacci polynomials $F_{n}(r)$;

2. Taking $a=2$ and $b=p=q=1$, we attain the Lucas polynomials $L_{n}(r)$;

3. Taking $a=q=1$ and $b=p=2$, we have the Pell polynomials $P_{n}(r)$;

4. Taking $a=b=p=2$ and $q=1$, we get the Pell-Lucas polynomials $Q_{n}(r)$;

5. Taking $a=b=1, p=2$ and $q=-1$, we obtain the Chebyshev polynomials $T_{n}(r)$ of the first kind;

6. Taking $a=1, b=p=2$ and $q=-1$, we have the Chebyshev polynomials $U_{n}(r)$ of the second kind.

These polynomials, the families of orthogonal polynomials and other special polynomials, as well as their generalizations, are potentially important in a variety of disciplines in many of sciences, specially in the mathematics, statistics and physics. For more information associated with these polynomials see [13, 14, 17, 18].

The generating function of the Horadam polynomials $h_{n}(r)$ (see [15]) is given by

$$
\Pi(r, z)=\sum_{n=1}^{\infty} h_{n}(r) z^{n-1}=\frac{a+(b-a p) r z}{1-p r z-q z^{2}} .
$$

Srivastava et al. [28] have studied the Horadam polynomials in a similar context involving analytic and bi-univalent functions, it was followed by such works as those by Al-Amoush [3], Wanas and Alb Lupaş [43] Abirami et al. [1] and others (see, for example, [4, 5, 20, 32, 33, 39, 45]).

In this paper we define a subclass $\mathcal{G}_{\Sigma}(\delta, \gamma, \lambda, \tau, r)$ of normalized analytic and biunivalent function using Borel distribution and Horadam polynomial $h_{n}(r)$. We obtain TaylorMaclaurin coefficient inequalities for functions belonging to the defined subclass $\mathcal{G}_{\Sigma}(\delta, \gamma, \lambda, \tau, r)$ and study the famous Fekete-Szegö problem.

\section{Main Results}

We begin this section by defining the family $\mathcal{G}_{\Sigma}(\delta, \gamma, \lambda, \tau, r)$ as follows:

Definition 2.1. For $0 \leq \delta \leq 1, \gamma \geq 0, \lambda \geq 1$, $0<\tau \leq 1$ and $r \in \mathbb{R}$, a function $f \in \bar{\Sigma}$ is said to be in the family $\mathcal{G}_{\Sigma}(\delta, \gamma, \lambda, \tau, r)$ if it satisfies the 
subordinations:

$$
\begin{gathered}
(1-\delta) \frac{z^{1-\gamma}\left(\mathcal{B}_{\tau} f(z)\right)^{\prime}}{\left(\mathcal{B}_{\tau} f(z)\right)^{1-\gamma}}+\delta \frac{z\left(\left(\mathcal{B}_{\tau} f(z)\right)^{\prime}\right)^{\lambda}}{\mathcal{B}_{\tau} f(z)} \\
\prec \Pi(r, z)+1-a
\end{gathered}
$$

and

$$
\begin{gathered}
(1-\delta) \frac{w^{1-\gamma}\left(\mathcal{B}_{\tau} g(w)\right)^{\prime}}{\left(\mathcal{B}_{\tau} g(w)\right)^{1-\gamma}}+\delta \frac{w\left(\left(\mathcal{B}_{\tau} g(w)\right)^{\prime}\right)^{\lambda}}{\mathcal{B}_{\tau} g(w)} \\
\prec \Pi(r, w)+1-a
\end{gathered}
$$

where $a$ is real constant and the function $g=f^{-1}$ is given by (2) .

Note: $\theta=(1-\delta)(\gamma+1)+\delta(2 \lambda-1)$ is used throyghout the paper unless othewise mentioned.

Theorem 2.1. For $0 \leq \delta \leq 1, \gamma \geq 0, \lambda \geq 1$, $0<\tau \leq 1$ and $r \in \mathbb{R}$, let $f \in \mathcal{A}$ be in the family $\mathcal{G}_{\Sigma}(\delta, \gamma, \lambda, \tau, r)$. Then

$$
\left|a_{2}\right| \leq \frac{e^{\tau}|b r| \sqrt{2|b r|}}{\sqrt{\left|[\varphi(\delta, \gamma, \lambda, \tau) b-2 p \theta] b r^{2}-2 q a \theta^{2}\right|}}
$$

and

$$
\left|a_{3}\right| \leq \frac{e^{2 \tau}|b r|}{\tau[(1-\delta)(\gamma+2)+\delta(3 \lambda-1)]}+\frac{e^{2 \tau} b^{2} r^{2}}{\theta^{2}},
$$

where

$$
\begin{gathered}
\varphi(\delta, \gamma, \lambda, \tau)=(1-\delta)(\gamma+2)(4 \tau+\gamma-1) \\
+2 \delta(2 \tau(3 \lambda-1)+2 \lambda(\lambda-2)+1)
\end{gathered}
$$

Proof Let $f \in \mathcal{G}_{\Sigma}(\delta, \gamma, \lambda, \tau, r)$. Then there are two analytic functions $u, v: U \longrightarrow U$ given by

$$
u(z)=u_{1} z+u_{2} z^{2}+u_{3} z^{3}+\cdots \quad(z \in U)
$$

and

$$
v(w)=v_{1} w+v_{2} w^{2}+v_{3} w^{3}+\cdots \quad(w \in U),
$$

with $u(0)=v(0)=0,|u(z)|<1,|v(w)|<1$, $z, w \in U$ such that

$$
\begin{gathered}
(1-\delta) \frac{z^{1-\gamma}\left(\mathcal{B}_{\tau} f(z)\right)^{\prime}}{\left(\mathcal{B}_{\tau} f(z)\right)^{1-\gamma}}+\delta \frac{z\left(\left(\mathcal{B}_{\tau} f(z)\right)^{\prime}\right)^{\lambda}}{\mathcal{B}_{\tau} f(z)}= \\
\Pi(r, u(z))+1-a
\end{gathered}
$$

and

$$
(1-\delta) \frac{w^{1-\gamma}\left(\mathcal{B}_{\tau} g(w)\right)^{\prime}}{\left(\mathcal{B}_{\tau} g(w)\right)^{1-\gamma}}+\delta \frac{w\left(\left(\mathcal{B}_{\tau} g(w)\right)^{\prime}\right)^{\lambda}}{\mathcal{B}_{\tau} g(w)}=
$$

$$
\Pi(r, v(w))+1-a .
$$

Or, equivalently

$$
(1-\delta) \frac{z^{1-\gamma}\left(\mathcal{B}_{\tau} f(z)\right)^{\prime}}{\left(\mathcal{B}_{\tau} f(z)\right)^{1-\gamma}}+\delta \frac{z\left(\left(\mathcal{B}_{\tau} f(z)\right)^{\prime}\right)^{\lambda}}{\mathcal{B}_{\tau} f(z)}=
$$

$$
1+h_{1}(r)+h_{2}(r) u(z)+h_{3}(r) u^{2}(z)+\cdots
$$

and

$$
\begin{aligned}
& (1-\delta) \frac{w^{1-\gamma}\left(\mathcal{B}_{\tau} g(w)\right)^{\prime}}{\left(\mathcal{B}_{\tau} g(w)\right)^{1-\gamma}}+\delta \frac{w\left(\left(\mathcal{B}_{\tau} g(w)\right)^{\prime}\right)^{\lambda}}{\mathcal{B}_{\tau} g(w)}= \\
& 1+h_{1}(r)+h_{2}(r) v(w)+h_{3}(r) v^{2}(w)+\cdots .
\end{aligned}
$$

Combining (6), (7), (8) and (9) yields

$$
(1-\delta) \frac{z^{1-\gamma}\left(\mathcal{B}_{\tau} f(z)\right)^{\prime}}{\left(\mathcal{B}_{\tau} f(z)\right)^{1-\gamma}}+\delta \frac{z\left(\left(\mathcal{B}_{\tau} f(z)\right)^{\prime}\right)^{\lambda}}{\mathcal{B}_{\tau} f(z)}=
$$

$$
1+h_{2}(r) u_{1} z+\left[h_{2}(r) u_{2}+h_{3}(r) u_{1}^{2}\right] z^{2}+\cdots
$$

and

$$
\begin{gathered}
(1-\delta) \frac{w^{1-\gamma}\left(\mathcal{B}_{\tau} g(w)\right)^{\prime}}{\left(\mathcal{B}_{\tau} g(w)\right)^{1-\gamma}}+\delta \frac{w\left(\left(\mathcal{B}_{\tau} g(w)\right)^{\prime}\right)^{\lambda}}{\mathcal{B}_{\tau} g(w)}= \\
1+h_{2}(r) v_{1} w+\left[h_{2}(r) v_{2}+h_{3}(r) v_{1}^{2}\right] w^{2}+\cdots .
\end{gathered}
$$

It is quite well-known that if $|u(z)|<1$ and $|v(w)|<1, z, w \in U$, then

$$
\left|u_{i}\right| \leq 1 \text { and }\left|v_{i}\right| \leq 1 \text { forall } i \in \mathbb{N} .
$$

Comparing the corresponding coefficients in (10) and (11), after simplifying, we have

$$
\begin{gathered}
{[(1-\delta)(\gamma+1)+\delta(2 \lambda-1)] e^{-\tau} a_{2}=h_{2}(r) u_{1},} \\
2 \tau[(1-\delta)(\gamma+2)+\delta(3 \lambda-1)] e^{-2 \tau} a_{3}+ \\
{\left[\frac{1}{2}(1-\delta)(\gamma+2)(\gamma-1)+\delta(2 \lambda(\lambda-2)+1)\right] e^{-2 \tau} a_{2}^{2}} \\
=h_{2}(r) u_{2}+h_{3}(r) u_{1}^{2},
\end{gathered}
$$

$$
-[(1-\delta)(\gamma+1)+\delta(2 \lambda-1)] e^{-\tau} a_{2}=h_{2}(r) v_{1}
$$


and

$$
\begin{gathered}
2 \tau[(1-\delta)(\gamma+2)+\delta(3 \lambda-1)] e^{-2 \tau}\left(2 a_{2}^{2}-a_{3}\right)+ \\
{\left[\frac{1}{2}(1-\delta)(\gamma+2)(\gamma-1)+\delta(2 \lambda(\lambda-2)+1)\right] e^{-2 \tau} a_{2}^{2}} \\
=h_{2}(r) v_{2}+h_{3}(r) v_{1}^{2} .
\end{gathered}
$$

It follows from (13) and (15) that

$$
u_{1}=-v_{1}
$$

and

$$
2 \theta^{2} e^{-2 \tau} a_{2}^{2}=h_{2}^{2}(r)\left(u_{1}^{2}+v_{1}^{2}\right) .
$$

If we add (14) to $(16)$, we find that

$\varphi(\delta, \gamma, \lambda, \tau) e^{-2 \tau} a_{2}^{2}=h_{2}(r)\left(u_{2}+v_{2}\right)+h_{3}(r)\left(u_{1}^{2}+v_{1}^{2}\right)$,

where $\varphi(\delta, \gamma, \lambda, \tau)$ is given by (5).

Substituting the value of $u_{1}^{2}+v_{1}^{2}$ from (18) in the right hand side of (19), we deduce that

$$
a_{2}^{2}=\frac{e^{2 \tau} h_{2}^{3}(r)\left(u_{2}+v_{2}\right)}{h_{2}^{2}(r) \varphi(\delta, \gamma, \lambda, \tau)-2 h_{3}(r) \theta^{2}} .
$$

Further computations using (3), (12) and (20), we obtain

$$
\left|a_{2}\right| \leq \frac{e^{\tau}|b r| \sqrt{2|b r|}}{\sqrt{\left|\left[\varphi(\delta, \gamma, \lambda, \tau) b-2 p \theta^{2}\right] b r^{2}-2 q a \theta^{2}\right|}} .
$$

Next, if we subtract (16) from (14), we can easily see that

$$
\begin{gathered}
2 \tau[(1-\delta)(\gamma+2)+\delta(3 \lambda-1)] e^{-2 \tau}\left(a_{3}-a_{2}^{2}\right)= \\
h_{2}(r)\left(u_{2}-v_{2}\right)+h_{3}(r)\left(u_{1}^{2}-v_{1}^{2}\right) .
\end{gathered}
$$

In view of (17) and (18), we get from (21)

$$
\begin{gathered}
a_{3}=\frac{e^{2 \tau} h_{2}(r)\left(u_{2}-v_{2}\right)}{2 \tau[(1-\delta)(\gamma+2)+\delta(3 \lambda-1)]}+ \\
\frac{e^{2 \tau} h_{2}^{2}(r)\left(u_{1}^{2}+v_{1}^{2}\right)}{2 \theta^{2}} .
\end{gathered}
$$

Thus applying (3), we obtain

$$
\left|a_{3}\right| \leq \frac{e^{2 \tau}|b r|}{\tau[(1-\delta)(\gamma+2)+\delta(3 \lambda-1)]}+\frac{e^{2 \tau} b^{2} r^{2}}{\theta^{2}} .
$$

This completes the proof of Theorem 2.1.

In the next theorem, we discuss the FeketeSzegö problem for the family $\mathcal{G}_{\Sigma}(\delta, \gamma, \lambda, \tau, r)$.
Theorem 2.2. For $0 \leq \delta \leq 1, \gamma \geq 0, \lambda \geq 1$, $0<\tau \leq 1$ and $r, \mu \in \mathbb{R}$, let $f \in \mathcal{A}$ be in the family $\mathcal{G}_{\Sigma}(\delta, \gamma, \lambda, \tau, r)$. Then

$$
\left\{\begin{array}{c}
\frac{e^{2 \tau}|b r|}{\tau[(1-\delta)(\gamma+2)+\delta(3 \lambda-1)]} ; \\
\text { for }|\mu-1| \leq \frac{\left|\left[\varphi(\delta, \gamma, \lambda, \tau) b-2 p \theta^{2}\right] b r^{2}-2 q a \theta^{2}\right|}{2 \tau b^{2} r^{2}[(1-\delta)(\gamma+2)+\delta(3 \lambda-1)]}, \\
\frac{2 e^{2 \tau}|b r|^{3}|\mu-1|}{\llbracket\left[\varphi(\delta, \gamma, \lambda, \tau) b-2 p \theta^{2}\right] b r^{2}-2 q a \theta^{2} \mid} ; \\
\text { for }|\mu-1| \geq \frac{\left|\left[\varphi(\delta, \gamma, \lambda, \tau) b-2 p \theta^{2}\right] b r^{2}-2 q a \theta^{2}\right|}{2 \tau b^{2} r^{2}[(1-\delta)(\gamma+2)+\delta(3 \lambda-1)]} .
\end{array}\right.
$$

Proof It follows from (20) and (21) that

$$
\begin{aligned}
a_{3}-\mu a_{2}^{2} & =\frac{e^{2 \tau} h_{2}(r)\left(u_{2}-v_{2}\right)}{2 \tau[(1-\delta)(\gamma+2)+\delta(3 \lambda-1)]}+(1-\mu) a_{2}^{2} \\
& =\frac{e^{2 \tau} h_{2}(r)\left(u_{2}-v_{2}\right)}{2 \tau[(1-\delta)(\gamma+2)+\delta(3 \lambda-1)]} \\
& +\frac{e^{2 \tau} h_{2}^{3}(r)\left(u_{2}+v_{2}\right)(1-\mu)}{h_{2}^{2}(r) \varphi(\delta, \gamma, \lambda, \tau)-2 h_{3}(r) \theta^{2}} \\
& =h_{2}(r)\left[\left(\psi(\mu, r)+\frac{e^{2 \tau}}{2 \tau[(1-\delta)(\gamma+2)+\delta(3 \lambda-1)]}\right.\right. \\
& \left.+\left(\psi(\mu, r)-\frac{e^{2 \tau}}{2 \tau[(1-\delta)(\gamma+2)+\delta(3 \lambda-1)]}\right) v_{2}\right]
\end{aligned}
$$

where

$$
\psi(\mu, r)=\frac{e^{2 \tau} h_{2}^{2}(r)(1-\mu)}{h_{2}^{2}(r) \varphi(\delta, \gamma, \lambda, \tau)-2 h_{3}(r) \theta^{2}} .
$$

According to (3), we find that

$$
\begin{gathered}
\left|a_{3}-\mu a_{2}^{2}\right| \leq \\
\left\{\begin{array}{l}
\frac{e^{2 \tau}|b r|}{\tau[(1-\delta)(\gamma+2)+\delta(3 \lambda-1)]} ; 0 \leq|\psi(\mu, r)| \leq \frac{e^{2 \tau}}{2 \tau[(1-\delta)(\gamma+2)+\delta(3 \lambda-1)]} \\
2|b r||\psi(\mu, r)| ; \quad|\psi(\mu, r)| \geq \frac{e^{2 \tau}}{2 \tau[(1-\delta)(\gamma+2)+\delta(3 \lambda-1)]} .
\end{array}\right.
\end{gathered}
$$

After some computations, we obtain

$$
\left|a_{3}-\mu a_{2}^{2}\right| \leq
$$

$$
\left\{\begin{array}{l}
\frac{e^{2 \tau}|b r|}{\tau[(1-\delta)(\gamma+2)+\delta(3 \lambda-1)]} ; \\
\quad \text { or }|\mu-1| \leq \frac{\left|\left[\varphi(\delta, \gamma, \lambda, \tau) b-2 p \theta^{2}\right] b r^{2}-2 q a \theta^{2}\right|}{2 \tau b^{2} r^{2}[(1-\delta)(\gamma+2)+\delta(3 \lambda-1)]} \\
\frac{2 e^{2 \tau}|b r|^{3}|\mu-1|}{\pi\left[\varphi(\delta, \gamma, \lambda, \tau) b-2 p \theta^{2}\right] b r^{2}-2 q a \theta^{2} \mid} ; \\
\text { for }|\mu-1| \geq \frac{\left|\left[\varphi(\delta, \gamma, \lambda, \tau) b-2 p \theta^{2}\right] b r^{2}-2 q a \theta^{2}\right|}{2 \tau b^{2} r^{2}[(1-\delta)(\gamma+2)+\delta(3 \lambda-1)]}
\end{array} .\right.
$$


Putting $\mu=1$ in Theorem 2, we obtain the following result:

Corollary 2.1. For $0 \leq \delta \leq 1, \gamma \geq 0, \lambda \geq 1$, $0<\tau \leq 1$ and $r \in \mathbb{R}$, let $f \in \mathcal{A}$ be in the family $\mathcal{G}_{\Sigma}(\delta, \gamma, \lambda, \tau, r)$. Then

$$
\left|a_{3}-a_{2}^{2}\right| \leq \frac{e^{2 \tau}|b r|}{\tau[(1-\delta)(\gamma+2)+\delta(3 \lambda-1)]} .
$$

\section{Conclusion}

The fact that we can find many unique and effective uses of a large variety of interesting functions and specific polynomial in Geometric Function Theory provided the primary inspiration for our analysis in this article. The primary objective was to create a new family $\mathcal{G}_{\Sigma}(\delta, \gamma, \lambda, \tau, r)$ of normalized analytic and bi-univalent function defined by Borel distribution and also using the Horadam polynomial $h_{n}(r)$, which are given by the recurrence relation (3) and generating function $\Pi(r, z)$ in (4). We generate Taylor-Maclaurin coefficient inequalities for functions belonging to this newly introduced bi-univalent function family $\mathcal{G}_{\Sigma}(\delta, \gamma, \lambda, \tau, r)$ and viewed the famous FeketeSzegö problem.

\section{References:}

[1] C. Abirami, N. Magesh and J. Yamini, Initial bounds for certain classes of bi-univalent functions defined by Horadam polynomials, Abstr. Appl. Anal., 2020, (2020), Article ID 7391058, 1-8.

[2] E. A. Adegani, S. Bulut and A. A. Zireh, Coefficient estimates for a subclass of analytic biunivalent functions, Bull. Korean Math. Soc., 55(2), (2018), 405-413.

[3] A. G. Al-Amoush, Coefficient estimates for certain subclass of bi functions associated with the Horadam Polynomials, arXiv:1812.10589v1, (2018), 1-7.

[4] A. G. Al-Amoush, Certain subclasses of biunivalent functions involving the Poisson distribution associated with Horadam polynomials, Malaya J. Mat., 7, (2019), 618-624.

[5] A. G. Al-Amoush, Coefficient estimates for a new subclasses of $\lambda$-pseudo biunivalent functions with respect to symmetrical points associated with the Horadam Polynomials, Turk. J. Math., 43, (2019), 2865-2875.

[6] Ş. Altınkaya and S. Yalçin, Poisson distribution series for certain subclasses of starlike functions with negative coefficients, $A n$ nals of Oradea University Mathematics Fascicola, 24(2), (2017), 5-8.
[7] K. O. Babalola, On $\lambda$-Pseudo-Starlike Functions, J. Class. Anal., 3(2), (2013), 137-147.

[8] S. Bulut, Coefficient estimates for general subclasses of m-fold symmetric analytic biunivalent functions, Turk. J. Math., 40, (2016), 1386-1397.

[9] S. Bulut and A. K. Wanas, Coefficient estimates for families of bi-univalent functions defined by Ruscheweyh derivative operator, Math. Moravica, 25(1), (2021), 71-80.

[10] P. L. Duren, Univalent Functions, Grundlehren der Mathematischen Wissenschaften, Band 259, Springer Verlag, New York, Berlin, Heidelberg and Tokyo, 1983.

[11] S. M. El-Deeb, T. Bulboaca and J. Dziok, Pascal distribution series connected with certain subclasses of univalent functions, Kyungpook Math. J., 59(2), (2019), 301-314.

[12] H. O. Güney, G. Murugusundaramoorthy and J. Sokół, Subclasses of bi-univalent functions related to shell-like curves connected with Fibonacci numbers, Acta Univ. Sapientiae, Mathematica, 10(1)(2018), 70-84.

[13] A. F. Horadam, Jacobsthal Representation Polynomials, The Fibonacci Quarterly, 35(2), (1997), 137-148.

[14] A. F. Horadam and J. M. Mahon, Pell and Pell-Lucas polynomials, The Fibonacci Quarterly, 23(1), (1985), 7-20.

[15] T. Hörçum and E. G. Kocer, On some properties of Horadam polynomials, Int. Math. Forum, 4, (2009), 1243-1252.

[16] S. Joshi, S. Joshi and H. Pawar, On some subclasses of bi-univalent functions associated with pseudo-starlike functions, J. Egyptian Math. Soc., 24, (2016), 522-525.

[17] T. Koshy, Fibonacci and Lucas Numbers with Applications, A Wiley- Interscience Publication, New York, 2001.

[18] A. Lupas, A Guide of Fibonacci and Lucas Polynomials, Octagon Mathematics Magazine, 7(1), (1999), 2-12.

[19] N. Magesh and S. Bulut, Chebyshev polynomial coefficient estimates for a class of analytic bi-univalent functions related to pseudostarlike functions, Afr. Mat., 29 (2018), 203209. 
[20] N. Magesh, J. Yamini and C. Abirami, Initial bounds for certain classes of bi-univalent functions defined by Horadam Polynomials, arXiv:1812.04464v1, (2018), 1-14.

[21] S. S. Miller and P. T. Mocanu, Differential Subordinations: Theory and Applications, Series on Monographs and Textbooks in Pure and Applied Mathematics Vol. 225, Marcel Dekker Inc., New York and Basel, 2000.

[22] W. Nazeer, Q. Mehmood, S. M. Kang and A. U. Haq, An application of Binomial distribution series on certain analytic functions, $J$. Comput. Anal. Appl., 26, (2019), 11-17.

[23] Á. O. Páll-Szabó and A. K. Wanas, Coefficient estimates for some new classes of biBazilevič functions of Ma-Minda type involving the Sălăgean integro-differential operator, Quaestiones Mathematicae, 44(4), (2021), 495502 .

[24] S. Porwal and M. Kumar, A unified study on starlike and convex functions associated with Poisson distribution series, Afr. Mat., 27, (2016), 10-21.

[25] T. G. Shaba and A. K. Wanas, Coefficient bounds for a new family of bi-univalent functions associated with $(\mathrm{U}, \mathrm{V})$-Lucas polynomials, Int. J. Nonlinear Anal. Appl., 13(1), (2022), 615-626.

[26] R. Singh, On Bazilevič functions, Proc. Amer. Math. Soc., 38(2),(1973), 261-271.

[27] H. M. Srivastava, Ş. Altinkaya and S. Yalçın, Hankel determinant for a subclass of biunivalent functions defined by using a symmetric q-derivative operator, Filomat 32, (2018), 503-516.

[28] H. M. Srivastava, Ş. Altınkaya and S. Yalçin, Certain subclasses of bi-univalent functions associated with the Horadam polynomials, Iran. J. Sci. Technol. Trans. A Sci., 43, (2019), 1873-1879.

[29] H. M. Srivastava, A. K. Mishra and P. Gochhayat, Certain Subclasses of Analytic and Bi-Univalent Functions, Appl. Math. Lett., 23, (2010), 1188-1192.

[30] H. M. Srivastava and A. K. Wanas, Initial Maclaurin coefficient bounds for new subclasses of analytic and m-fold symmetric biunivalent functions defined by a linear combination, Kyungpook Math. J., 59(3), (2019), 493-503.
[31] H. M. Srivastava, A. K. Wanas and H. Ö. Güney, New families of bi-univalent functions associated with the Bazilevič functions and the $\lambda$-Pseudo-starlike functions, Iran. J. Sci. Technol. Trans. A: Sci., 45, (2021), 1799-1804.

[32] H. M. Srivastava, A. K. Wanas and G. Murugusundaramoorthy, Certain family of biunivalent functions associated with Pascal distribution series based on Horadam polynomials, Surveys Math. its Appl., 16, (2021), 193205.

[33] H. M. Srivastava, A. K. Wanas and R. Srivastava, Applications of the $q$-SrivastavaAttiya operator involving a certain family of bi-univalent functions associated with the Horadam polynomials, Symmetry, 13, (2021), Art. ID 1230, 1-14.

[34] S. R. Swamy, P. K. Mamatha, N. Magesh and J. Yamini, Certain subclasses of bi-univalent funtions defined by Sălăgean operator associated with the $(p, q)$ - Lucas polynomials, Advances in Mathematics, Scientific Journal, 9(8), (2020), 6017-6025.

[35] S. R. Swamy, J. Nirmala and Y. Sailaja, Some special families of holomorphic and AiOboudi type bi-univalent functions associated with $(\mathrm{m}, \mathrm{n})$-Lucas polynomials involving modified sigmoid activation function, South East Asian J. of Mathematics and Mathematical Sciences, 17(1), (2021), 01-16.

[36] S. R. Swamy, A. K. Wanas and Y. Sailaja, Some special families of holomorphic and Sălăgean type bi-univalent functions associated with (m,n)-Lucas polynomials, Communications in Mathematics and Applications, 11(4), (2020), 563-574.

[37] A. K. Wanas, Applications of $(\mathrm{M}, \mathrm{N})$-Lucas polynomials for holomorphic and bi-univalent functions, Filomat, 34, (2020), 3361-3368.

[38] A. K. Wanas, Coefficient estimates for Bazilevič functions of bi-Prestarlike functions, Miskolc Mathematical Notes, 21(2), (2020), 1031-1040.

[39] A. K. Wanas, Horadam polynomials for a new family of $\lambda$-pseudo bi-univalent functions associated with Sakaguchi type functions, Afr. Mat., 32, (2021), 879-889.

[40] A. K. Wanas and N. A. Al-Ziadi, Applications of Beta negative binomial distribution series on holomorphic functions, Earthline J. Math. Sci., 6(2), (2021), 271-292. 
[41] A. K. Wanas and L.-I. Cotîrlǎ, Initial coefficient estimates and Feketeâ" Szegö inequalities for new families of bi-univalent functions governed by (p-q)-Wanas operator, Symmetry, 13, (2021), Art. ID 2118, 1-17.

[42] A. K. Wanas and J. A. Khuttar, Applications of Borel distribution series on analytic functions, Earthline J. Math. Sci., 4, (2020), 71-82.

[43] A. K. Wanas and A. Alb Lupas, Applications of Horadam polynomials on Bazilevič biunivalent function satisfying subordinate conditions, Journal of Physics: Conf. Series, 1294, (2019), 1-6.

[44] A. K. Wanas and A. H. Majeed, On subclasses of analytic and $m$-fold symmetric bi-univalent functions, Iranian J. Math. Sci. Inform., 15(2), (2020), 51-60.

[45] A. k. Wanas and S. Yalçin, Horadam polynomials and their applications to new family of bi-univalent functions with respect to symmetric conjugate points, Proyecciones (Antofagasta, On line), 40, (2021), 107-116.

\section{Contribution of individual authors to the creation of a scientific article (ghostwriting policy)}

S. R. Swamy - conceptualization, methodology, formal analysis.
Alina Alb Lupaş - writing - review and editing, supervision, funding acquisition.

Abbas Kareem Wanas - software, validation, data curation, writing - original draft preparation, project administration.

J. Nirmala - investigation, resources, visualization.

All authors have read and agreed to the published version of the manuscript.

Follow: www.wseas.org/multimedia/contributorrole-instruction.pdf

\section{Sources of funding for research presented in a scientific article or scientific article itself}

Report potential sources of funding if there is any

\section{Creative Commons Attribution \\ License 4.0 (Attribution 4.0 \\ International , CC BY 4.0)}

This article is published under the terms of the Creative Commons Attribution License 4.0

https://creativecommons.org/licenses/by/4.0/deed.en_US 